\title{
Die enthemmte Serie: Überlegungen zu Hemmung und Enthemmung am Beispiel von Game of Thrones.
}

\author{
Merve Winter, Timo Storck und Jelka Berger (Berlin)
}

Zusammenfassung: Zeitgenössische TV-Serien loten zunehmend die Grenzen des Darstellbaren aus und überschreiten diese, was das Zeigen von gewaltvollen Szenen angeht. Besonders die erfolgreiche Fantasy-Serie Game of Thrones (im Weiteren: GoT) ist hier zu nennen, die wir in dem vorliegenden Beitrag unter dem Blickwinkel von Hemmung und Enthemmung betrachten wollen. Dafür untersuchen wir diese Begriffe zunächst in der Freudschen Verwendung, wo wir Hemmung einerseits im Kontext des Über-Ichs, im Sekundärprozess als Hemmung des Primärprozesses, und schließlich in der Kultur als Ergebnis eines Triebverzichts finden. Diese Freudschen Überlegungen wollen wir nutzen, um die Rezeption von GoT zu verstehen. Dabei folgen wir der Annahme, dass sich in medialen Produkten immer auch das Sag- und Zeigbare einer Gesellschaft spiegelt und sich somit Aussagen über deren Verfasstheit ableiten lassen. Eine psychoanalytische Untersuchung ausgewählter Episoden, bei der den Irritationen und freien Einfällen der Rezipierenden gefolgt wird, verdeutlicht, dass GoT gesellschaftlich virulente Gefühle inszeniert, damit aber auch greifbar und bearbeitbar macht. So wird den Zuschauenden ein Gefühl eines Mangels an Sicherheit vermittelt, das sich als Spiegelung eines in den westlichen Industriegesellschaften verbreiteten Lebensgefühls seit 9/11 verstehen lässt. Des Weiteren werden Bedingungen zum moralischen Handeln und ethischen Empfinden und dabei auch die Rolle und Bedeutung der Familie hinterfragt. Bei den Zuschauenden führt die Rezeption zu einer interpassiven Verschränkung von (abgewehrten) lustvollen und unlustvollen Aspekten angesichts der äußeren und inhaltlichen Grausamkeit.

Schlüsselwörter: Gewalt, TV-Serien, Game of Thrones, Hemmung, Enthemmung 


\section{$1 \quad$ Einleitung}

Wer sich zeitgenössische TV-Serien wie Game of Thrones (HBO, 2011-2019), Vikings (History Television, 2013-), Sons of Anarchy (FX Network, 2008-2014) oder The Walking Dead (AMC, 2010-) anguckt, muss hart im Nehmen sein: Menschen werden auf alle erdenklichen Arten gefoltert und getötet, es wird lebend kastriert, amputiert und vergewaltigt. Dabei wird auch vor Kindern nicht haltgemacht. Babies werden getötet, Kinder lebend ans Kreuz genagelt oder auf dem Scheiterhaufen verbrannt. Die Grenzen des Sag- und Zeigbaren haben sich in der massentauglichen Film- und Serienindustrie offenbar verschoben. Es liegt nahe, hier an eine Enthemmung zu denken, die in den Mainstream eingezogen ist. Wo früher die sogenannten «Splatter-Filme» ein Nischendasein in den Videotheken führten und man (von wenigen Ausnahmen mal abgesehen) noch vergleichsweise sicher davon ausgehen konnte, von sehr grausamen oder blutigen Szenen, bzw. dem Tod der Hauptdarstellerinnen ${ }^{1}$ in Kino oder TV-Serie nicht überrascht zu werden, sind heute die Grenzen zwischen dem «Splatter-» oder «Horror-Genre» und dem Mainstream-Kino, bzw. der TV-Serie weniger erkennbar. Zwar gibt es Serien wie Mad Men (AMC, 2007-2015), The Deuce (HBO, 2017-), Homeland (Showtime, 2011-2019), Masters of Sex (Showtime, 2013-2016) und viele mehr, die ohne solche grausamen Szenen auskommen, aber die Zeiten von Unsere kleine Farm (NBC, 1974-1984), Lassie (CBS, 1954-1973), Dr. Quinn (CBS, 1993-1998), oder Ein Colt für alle Fälle (ABC, 1981-1986) sind wohl vorbei (auch wenn es seit kurzem eine- wenig euphorisch besprochene - Neuauflage von McGyver gibt). Im vorliegenden Beitrag wollen wir der Frage nachgehen, welche Funktion diese von uns wahrgenommene Enthemmung im Zeigen von grausamen Szenen hat, bzw. ob sich überhaupt eine solche Funktion benennen lässt. Dabei hat uns interessiert, ob diese Serien sich trotz der zur Schau gestellten Gewalt einer solchen Beliebtheit erfreuen oder gerade wegen dieser, eine Frage, die sich im vorliegenden Kontext nur hypothetisch bzw. konzeptuell beantworten lässt. Die Diskussion um die Enthemmung kreuzt dabei unvermeidlich die Frage nach der allgemeinen Funktion von Gewalt in Film und Fernsehen, wozu es etliche Veröffentlichungen gibt, die hier nur ausschnittartig zur Kenntnis genommen werden können. Ähnliches gilt für die Sexualität, bei der wir interessanterweise weniger von einer Enthemmung sprechen können: Zwar sind Kino und Fernsehen durchaus freizügiger geworden, doch die Grenzen zwischen der Pornoindustrie und dem Mainstream-Fernsehen scheinen weiterhin zu bestehen. Speziell das US-amerikanische Fernsehen und Kino tut sich nach wie vor schwer mit allzu expliziten Sexszenen, das Zeigen von Vaginas oder erigierten Penissen ist nach wie vor tabu. Das gilt auch für die Serie Game of Thrones, obwohl gerade diese 
für ihre freizügigen Sexszenen bekannt ist. Mit rund 30 Millionen Zuschauenden (Schröder, 2017) - die illegalen Downloads noch nicht eingerechnet - ist GoT die am häufigsten gesehene Serie überhaupt, und erfüllt - ebenso wie die oben genannten Serien - die Kriterien, die nach Winter (2013, S. 75) eine «Kultserie» ausmachen: «Polysemie, Offenheit und Widersprüche», die zu «Gesprächen und Diskussionen unter den Kultisten [führen], die gemeinsame Bande verstärken und aus ihnen eine interpretative Gemeinschaft machen.». Kultserien basieren nach Winter zudem auf der «Dekonstruktion vertrauter Erwartungen» und legitimieren abweichende, nicht normale Praktiken, wodurch sie «Spannungen, soziale Probleme und Konflikte in der Gesellschaft» reflektieren (a.a. O., S. 74). Gerade GoT ist zudem für seine äusserst brutalen Szenen bekannt und bricht mit vertrauten Erwartungen in derWeise, dass in dieser Serie in einem bis dahin nicht gekannten Ausmass Hauptdarsteller sterben.

Vor diesem Hintergrund möchten wir uns im Folgenden aus einer kulturwissenschaftlich-psychoanalytischen Perspektive den zeitgenössischen TV-Serien, insbesondere GoT zuwenden, mit der Annahme, dass sich in Filmen und Serien immer etwas vom Sag- und Zeigbaren der jeweiligen Zeit zeigt und diese Medien so gesehen zu einem Gradmesser für die Verfasstheit der Gesellschaft gerade auch im Hinblick auf Hemmung bzw. Enthemmung werden können (vgl. Winter, M., Storck, T. et al., 2018). Doch was kann überhaupt unter diesen Begriffen verstanden werden?

\section{$2 \quad$ Hemmung und Enthemmung in Freudscher Perspektive}

Wer sich den Begriffen Hemmungund Enthemmungaus psychoanalytischer Perspektive nähern will, stösst schnell auf das Problem, dass der eine Begriff primär in einem Text, der andere von Freud gar nicht thematisiert wird. Im Folgenden blicken wir zunächst auf die wenigen Bemerkungen Freuds zur Hemmung und die impliziten Folgerungen für ein Verständnis von Enthemmung. Dabei wird vor allem das Instanzenmodell aus Ich, Es und Über-Ich die entscheidende Referenz sein.

\subsection{Hemmung im Instanzenmodell des Psychischen}

In der Arbeit Hemmung, Symptom und Angstvon 1926 betont Freud zunächst, dass die Unterscheidung zwischen Hemmungen und (psychischen) Symptomen zumindest im alltäglichen Sprachgebrauch unscharf sei, bei genauerer Hinsicht aber deutlich werde, dass die Hemmung gegenüber dem Symptom das weniger schwere Problem sei und nicht notwendigerweise etwas Pathologisches bedeuten müsse. Eine Hemmung ist nach Freud zunächst einmal eine einfache Herabsetzung 
der Ich-Funktion, bzw. eine «Funktionseinschränkung des Ichs» (Freud, 1926d, S. $116 \mathrm{f}$.), die sehr verschiedene Ursachen haben kann. Entweder entsteht eine solche Funktionseinschränkung (z. B. im sexuellen Bereich) um einem Konflikt mit dem Es auszuweichen, oder (etwa im beruflichen Bereich) um nicht in Konflikt mit dem Über-Ich zu geraten (a.a.O., S. 236), oder aber infolge von (in Freuds libidotheoretischen Worten gesprochen) «Energieverarmung». Sehr viel mehr ist von Freud nicht zum Begriff der Hemmung zu erfahren, fortan diskutiert er diesen vor allem in seiner symptomhaften Ausprägung, also als Hemmung, die sich in der Folge unbewältigter Wunsch-Abwehr-Konflikte einstellt. Die Enthemmung muss dem gegenüber primär als Gegenteil der Hemmung verstanden werden. Wenn eine Hemmung wie in Hemmung, Symptom und Angst eine Funktionseinschränkung des Ichs bedeutet, oder - wie im Symptom - gänzlich unbewusst abläuft, so könnte man zunächst davon ausgehen, dass eine Enthemmung etwas Positives darstellt: Wenn etwas oder jemand enthemmt ist, ist er/es zumindest nicht ge-hemmt. Und in dieser positiven Konnotation macht sich beispielsweise die Sexindustrie den Begriff auch zu eigen. Nun hat es die Wortbedeutung aber in sich, dass mit Enthemmung gemeinhin auch ein Überschiessen bezeichnet wird. Wer enthemmt ist, leidet, so könnte vermutet werden, nicht bloss unter einer Funktionseinschränkung des Ichs, sondern an einer psychischen «Umgehung» der Über-Ich-Funktionen. Es gibt keine Grenzen und Gesetze mehr, das Es hat - bleibt man im Modell der psychischen Instanzen - die Herrschaft über das psychische Geschehen erlangt. Dass dies kein wünschenswerter Zustand ist, leuchtet schnell ein. Besonders anschaulich beschreibt Freud unter Rekurs auf Le Bon einen solchen Zustand bei den Massenindividuen, bei denen

, alle individuellen Hemmungen entfallen und alle grausamen, brutalen, destruktiven Instinkte, die als Überbleibsel der Urzeit im Einzelnen schlummern, zur freien Triebbefriedigung geweckt werden. (Freud, 1921c, S. 84)

Enthemmung wäre dann gleichzusetzen mit einer Regression auf eine frühere Stufe der Libidoentwicklung, wenn nicht gar der Menschheitsentwicklung an sich:

, Ferner steigt durch die bloße Zugehörigkeit zu einer organisierten Masse der Mensch mehrere Stufen auf der Leiter der Zivilisation herab. In seiner Vereinzelung war er vielleicht ein gebildetes Individuum, in der Masse ist er ein Barbar, das heißt ein Triebwesen (Le Bon zitiert nach Freud, 1921, S. 81 f.). 
Hier wird Freuds Konzeption «zielgehemmter» Triebregungen bedeutsam. Eine solche ist dann zielgehemmt, wenn sie eingebunden ist in zärtliche oder soziale Motive, etwa die Liebe zu den Eltern, Freundschaftsbeziehungen oder Gruppenzusammengehörigkeit. «[Z]ielgehemmte Triebe», so Freud, sind

, Triebregungen aus gut bekannten Quellen mit unzweideutigem Ziel. Die aber auf dem Weg zur Befriedigung haltmachen, so dass eine dauernde Objektbesetzung und eine anhaltende Strebung zustande kommt. (1933, S. 103)

Implizit enthalten ist darin die Figur einer eigentlichen Nicht-Gehemmtheit des Triebes, der als solcher eben nicht in soziale oder zärtliche Bindungen führt. Libidotheoretisch entscheidend ist hier, dass der Anreiz der Zielhemmung darin besteht, dass Befriedigung nicht mit einem einzelnen Akt der Triebbefriedigung «erledigt» ist, sondern dass «dauernde Bindungen» geschaffen werden, «während die direkt sexuellen jedesmal durch die Befriedigung ihrer Energie verlustig werden» (1921, S. 156). Hemmung in diesem Sinn meint die Voraussetzung für andere als momentane Lust/Befriedigung, also eine Art von permanentem Gefühl von Verbundenheit und Zugehörigkeit. Dass es mit solchen Gefühlen bei GoT nicht so weit her ist, werden wir weiter unten noch diskutieren.

Das, was die Kultur in einem langen und oftmals gewaltsamen Prozess der Disziplinierung errichtet hatte, wird also in einem enthemmten bzw. nicht (mehr) zielgehemmten Zustand aufgehoben. Marcuse zufolge gibt es aber insbesondere in einer Gesellschaft, in der die Aggression beispielsweise durch automatische Waffen technologisiert ist, durchaus ein Bedürfnis nach solchen primitiveren Formen der Aggression. Denn eine repressiv entsublimierte und technisierte Gesellschaft kann, so Marcuse, die primären Impulse nicht befriedigen und beschwichtigen, die umso mehr nach Wiederholung, Intensivierung und Eskalation drängen (Marcuse 1967, S. 466).

Marcuse betont, dass zwar der Gebrauch von Werkzeugen der Aggressivität so alt wie die Zivilisation selbst sei, aber zwischen den technologischen und den primitiveren Formen der Aggression ein grosser Unterschied bestehe:

Das Messer, das "plumpe Werkzeug» und sogar der Revolver sind in viel stärkerem Maße «Teil» des Individuums, welches sie benutzt, und sie bringen dieses Individuum in eine engere Beziehung zu seinem Ziel. Die menschlichen Opfer des Gewehrs sind wahrnehmbar, 
die des Bombenflugzeuges und der Rakete sind der Wahrnehmung des Täters entrückt. (Marcuse, 1967, S. 466)

Wir können also bereits an dieser Stelle die These aufstellen, dass beim Gucken von GoT möglicherweise genau diese primären Impulse nach Aggressivität bedient werden.

\subsection{Denken als Hemmung des Primärprozesses}

Die konzeptuellen Folgerungen aus Freuds Bemerkungen zum verschobenen Verhältnis der Instanzen in der Enthemmung sowie die Figur der (trieb-) zielgehemmten Kulturentwicklung haben bereits einen weiteren Bereich anklingen lassen, der eine implizite Grundstruktur von Freuds Konzeption von Hemmung/ Enthemmung bildet: das Verhältnis von Primär- und Sekundärprozess (vgl. in allgemeinpsychologischer Hinsicht Billhardt \& Storck, in Vorb.). Verkürzt gesprochen beschreibt die Konzeption, im hier relevanten Sinn, eine Grundstruktur primärprozesshaften psychischen Funktionierens, die sich v.a. dadurch auszeichnet, dass kein «Triebaufschub» möglich oder nötig ist. Der dem Lustprinzip verschriebene Primärprozess kennt keine lineare Zeitlichkeit, keine Negation - und vor allem keine realitätsorientierte Ausgestaltung von Befriedigungsformen (vgl. Freud, 1915b, S. 485 f.). Erst auf der Ebene des Sekundärprozess wird die (soziale) Realität einbezogen, spielen zeitliche Abfolgen eine Rolle und können Konsequenzen von Handlungen und Handlungsvorstellungen einbezogen werden. Hemmung wiederum kann dann verstanden werden als (relative) Unterbrechung von Erregungsabläufen (durch sogenannte «Seitenbesetzungen»; Freud, 1950) mit dem Ergebnis von Ablenkungen auf andere Vorstellungen und/oder einer Verringerung der Intensität von Reiz oder Erregung.

\subsection{Erregung versus Lust, Befriedigung}

Freuds in libidotheoretischer Sprache formulierte Konzeption gewinnt an Überzeugungskraft, wenn man sich vor Augen führt, dass der Verzicht auf die Hemmung von Erregungsabläufen, in welcher der Sekundärprozess besteht, zwar dem Lustprinzip folgt, aber nicht schlicht derart verstanden werden kann, dass er ausschliesslich erstrebenswert wäre. Bei Freud sind Lust und Erregung als Gegenspieler zu verstehen (das kann als einer der Grundpfeiler der psychoanalytischen Konflikttheorie gelten; Storck, 2018): Erregung bedeutet Reizung, Stimulierung. Lust (bzw. Befriedigung) ist die Empfindung, die vom Absinken einer 
Reizintensität hervorgerufen wird, der Anstieg der Intensität eines Reizes führt zum Erleben von Unlust (Freud, 1915c, S. 214 ff.). In dieser Perspektive streben wir nicht nach Erregung, sondern nach Befriedigung, und es muss angenommen werden, dass ab einem bestimmten Mass Erregung unlustvoll erlebt wird. Hemmung, ob nun beschrieben als vermittelt über die Instanz des Über-Ichs oder beschrieben als Aktivität des Sekundärprozesses, bedeutet dann Reglementierung von Erregung, schafft die Formen für Befriedigung (und das meist auf «sozialverträgliche» und emotionsdynamisch ertragbare Weise). Der Verzicht auf Hemmung setzt entsprechend das Lustprinzip (als Prinzip, das auf Lust/Befriedigung abzielt) paradoxerweise gerade ausser Kraft. Ent-hemmung meint also fortgesetzte Stimulierung, und damit Erregung, die kein Mass findet (und gerade nicht eine Sucht nach Befriedigung).

\section{$3 \quad$ Hemmung und Enthemmung im Kino und in TV-Serien}

Untersucht man die Entwicklung von Hemmung und Enthemmung am Beispiel der Medienlandschaft, so kommt man nicht um einen Blick auf die strukturellen Rahmenbedingungen des US-amerikanischen Fernsehens umhin, die sich grob in drei Phasen unterscheiden lassen (Eichner, 2013). Die erste Phase (ca. 1920er-1970er Jahre) wird durch die Vorherrschaft der drei grossen Networks NBC, CBS und ABC begründet und lässt serielle Formate wie beispielsweise The Dean Martin Show und auch Kriminal-, Anwaltsserien und Soap Operas entstehen. Qualitativ kann diese Phase als erstes goldenes Fernsehzeitalter beschrieben werden, welches mit den in den 1950er Jahren live ausgestrahlten Bühnenstücken begründet wird (vgl. Thompson, 1996). Die Filme, die in dieser ersten Phase entstehen, sind stark durch die Konventionen des sogenannten Production Code (oder auch Hay Code) geprägt. Dieses Regelwerk wurde 1930 von der von den grossen Hollywoodstudios gegründeten Regulierungsbehörde Motion Picture Producers and Distributors of America (MPPDA) beschlossen und trat 1934 auf Druck der katholischen Kirche verbindlich in Kraft (Black, 1989; Greene, 2010). Neben sexuellen Inhalten stand vor allem die Darstellung krimineller Akte im Mittelpunkt der Zensur. So legte der Code fest, dass eine kriminelle Figur, die andere materiell oder physisch schädigt, nicht als Held dargestellt, Personen des öffentlichen Dienstes wie Polizisten nicht zu Mordopfern, Waffen nur in begrenztem Masse gezeigt und Gewalttaten nicht explizit dargestellt werden dürfen. Zudem gäbe es kein Motiv - mit Ausnahme der Notwehr - das einen Mord rechtfertige (Timmer, 2011). Durch die Abschaffung des Production Code Ende der 1960er Jahre folgte der Bruch mit diesen, von der Filmindustrie selbstauferlegten, Konventionen oder 
besser: Hemmungen, und neben einem Boom von Horrorfilmen werden Filme wie Bonny and Clyde (1967), The Wild Bunch (1969), A Clockwork Orange (1979) oder The Godfather (1972) möglich (Thompson, 1996, S. 40 f.). Das sogenannte «Mitternachtskino» entstand, und die Kinobesitzer zeigten in Sonderprogrammen Exploitationsfilme, Horrorfilme und andere blutrünstige Machwerke wie Night oft the Living Dead (1968) oder Texas Chainsaw Massacre (1974) (Winter, 2013, S. 73). AusWesternfilmen war bisher zwar die Darstellung von Kampf- oder Gefechtsszenen bekannt, allerdings ohne die dadurch ausgelösten physischen Folgen zu dokumentieren (z. B. die Kugel, die die Brust trifft). Diese wurden nun explizit dargestellt und beispielsweise das Shoot-Out, also ein (meistens final) ausgetragenes Waffenduell, etabliert (Metelmann, 2008). Mit der Abschaffung des Production Code als einem Teil des gesellschaftlich-medialen Über-Ichs brachen sich bereits Ende der 60er Jahre also primitivere und archaischere Formen der Gewalt Bahn, was zunächst heftige gesellschaftliche Kontroversen auslöste. Als einer der ersten Filme, die das sogenannte Gewaltkino begründete, kann nach Winter The Wild Bunch gelten, der «durch seine Darstellung physischer Schmerzen und deformierter Leichen auch den visuellen Körperhorror der Splatterfilme vorweg[nahm]» (2015a, S. 156). Aber anders als für viele nachfolgende Filme war für den Regisseur Sam Peckinpah «das Zurschaustellen exzessiver Gewalt und von Brutalität [...] kein Selbstzweck»: Nach Winter war es das Ziel von Peckinpah, das Publikum «für Gewalt und deren schreckliche Folgen [zu] sensibilisieren» (a.a.O., 157). Dies geschah durch eine für damalige Verhältnisse enthemmte Darstellung, die die Grenzen des bislang Zeigbaren überschritt. Aber auch die heftige Kritik von kirchlichen Vereinigungen und laute Rufe nach Wiedereinführung einer Zensurbehörde konnten nicht verhindern, dass die zunehmenden Gewaltdarstellungen im Kino vom Kinopublikum immer mehr akzeptiert wurden und für die Medienbranche ein zunehmend gutes Geschäft waren (ebd. S. 159).

Das Aufkommen von Kabelsendern und der damit verbundene Verlust des Monopols der grossen Networks begründet die zweite Phase im US-amerikanischen Fernsehen, die ungefähr von Mitte der 70er Jahre bis Mitte der 90er Jahre reichte (Eichner, 2013). Es entstehen Pay-TV-Sender wie Home Box Office (HBO, seit 1972) und FOX (seit 1986) die sich dem Seriengenre innovativ nähern.

Inhaltlich sieht Thompson (1996) durch die Serie Hill Street Blues 1981 (MTM, 1981-1987; im deutschen Fernsehen ab 1985 als Polizeirevier Hill Street), das «zweite goldene Fernsehzeitalter» begründet (ebd. S. 30 ff.). Statt eines Hauptcharakters wie bislang üblich, gibt es nun mehrere, gleichberechtigte Figuren, deren Charaktere ambivalenter sind. Die klassische Heldenfigur ist genau wie cha- 
rakterliche schwarz-weiss-Malerei abgeschafft. Im Filmgenre entsteht in den 90er Jahre durch Regisseure wie Quentin Tarantino (z. B. Pulp Fiction, USA, 1994) oder Oliver Stone (z. B. Natural Born Killers, USA, 1994) eine neue Art der dargestellten Gewalt, die sich dadurch kennzeichnet, dass ihr keine erkennbare Motivation mehr zugrunde liegt (Metelmann, 2008): «Erst der unbefangene Mord stellt die ultimative Herausforderung und Tabuverletzung dar» (Zielcke, 1995). Denn «Gewalt, der nicht eine wie auch immer geartete Motivation zugeschrieben werden kann, verunsichert am meisten» (Metelmann, 2008, S. 119). Neben dem offenkundigen Mangel an Motiven ist es zudem eine neue Öffentlichkeit, in der der Mord stattfindet und stattfinden muss, um dem Täter dadurch Bedeutung und schliesslich auch Identität zu verleihen (Zielcke, 1995).

An diese Entwicklung lehnt sich die dritte Phase des US-amerikanischen Fernsehens ab circa Mitte der 90er Jahre, die durch zunehmende Digitalisierung und Globalisierung geprägt ist. Auch das Qualitätsfernsehen und damit HBO als einer dessen prominentester Produzenten (Eichner, 2013) kommt hier zu voller Blüte. Dabei profitiert HBO von seiner Natur als Pay-TV-Sender; es dürfen Dinge gezeigt werden, die im Free-TV schwer vorstellbar wären. So ist es auch der Sender HBO, der mit der Serie The Sopranos (HBO, 1999-2007) - genauer gesagt mit der fünften Episode der ersten Staffel - dem dritten goldenen Zeitalter die Tür öffnet (Gormász, 2015). In dieser Folge begeht Tony Soprano, einer der Hauptfiguren, einen Mord ohne Motiv und weitet damit auch für die Serienmacherinnen die Grenzen des Möglichen und Zeigbaren aus (Kalle, 2008). In diesem Geiste entstehen Serien wie The Wire (HBO, 2002-2008), Breaking Bad (AMC, 2008-2013) und schliesslich auch Game of Thrones. (HBO, 2011-2019)

\section{$4 \quad$ Methodik}

Als methodischem Referenzpunkt der vorliegenden Arbeit orientieren wir uns an der Filmpsychoanalyse (vgl. z. B. Hamburger, 2018), die sich im Kern als eine mediale Wirkungsanalyse bezeichnen lässt. Dabei wird insofern ein psychoanalytischer Zugang gewählt, als sich die Rezeption eines medialen Untersuchungsgegenstands, z. B. Film oder TV-Serie, als eine Beziehungssituation verstehen lässt, die auf ihre latenten Strukturen hin befragt werden kann. Dazu wird den freien Einfällen und Irritationen der Rezipierenden gefolgt (das spielt auch im Ansatz einer tiefenhermeneutischen Kulturanalyse Lorenzers, 1986, eine entscheidende Rolle). Auf konsequenteWeise wird dabei das mediale Produkt, und nur dieses, zum Gegenstand der Analyse, jeder Einbezug von Kontextinformationen 
überschreitet den methodischen Zugang. Ebenso wird nicht Theorie auf den Untersuchungsgegenstand angewendet, sondern eine Methode.

Im Weiteren werden wir also unsere Rezeption einer enthemmt wirkenden Darstellung von Gewalt in GoT, die unterstützt wird durch das Phänomen der medialen Wahrnehmung der Serie über ihre Gewaltszenen, z. B. in Foren, zum Ausgang nehmen. Dazu werden wir im Folgenden drei Szenen besprechen, die in solchen Foren besonders kontrovers besprochen wurden, um uns dann der Irritation angesichts dessen mittels eines Verstehensversuchs entlang psychoanalytischer Überlegungen zu Hemmung und Enthemmung zu nähern.

\section{$5 \quad$ Gewalt und Enthemmung in Game of Thrones}

Die TV-Serie Game of Thrones, eine Fantasy-Saga, die jedoch auch Elemente anderer Genres enthält, basiert auf der Romanreihe $A$ song of ice and fire von George R.R. Martin und wurde 2011 von David Benioff und Daniel B. Weiss für den Kabelsender HBO adaptiert. Die Handlung ist auf den fiktiven Kontinenten Westeros und Essos angesiedelt, und im Mittelpunkt der Geschichte steht der Kampf verschiedener Adelshäuser um die Macht (in Form des Eisernen Throns). Die Hauptcharaktere entstammen den Dynastien Stark, Baratheon, Lannister und Targaryen.

\section{1 «Baelor» (S1, E9)}

Ned Stark, der Lord von Winterfell und Wächter des Nordens, wird des Verrats am neuen König Joffrey Baratheon angeklagt. Öffentlich soll er seine Schuld gestehen und Joffrey als König anerkennen, was er (um seine Tochter zu schützen) schliesslich auch tut. Sowohl das Publikum innerhalb der Serie als auch das vor dem Fernsehbildschirm rechnet nun damit, dass er begnadigt wird. Doch Joffrey verweigert ihm die Gnade und verurteilt ihn zum Tode ("bring me his head"). Seine Töchter Arya und Sansa müssen tatenlos mitansehen, wie ihrVater enthauptet wird. Der Tod von Ned Stark schockiert, da der Zuschauende mit einem Bruch seiner sonstigen Sehgewohnheiten konfrontiert wird. Eine Hauptfigur, die zusätzlich als Sympathieträger aufgebaut wurde, stirbt (eigentlich) nicht. Zudem ist sein Kontrahent der jugendliche Joffrey Baratheon, der dem in sämtlichen Bereichen überlegenen «Wächter des Nordens» kein ebenbürtiger Gegner zu sein scheint. Dass ausgerechnet er ihn zu Fall bringt, vermittelt dem Zuschauenden: das Böse kann siegen, niemand ist sicher (vor niemandem). Der Fakt, dass Joffrey Ned tatsächlich enthaupten lässt, ist nicht nur für die Zuschauenden vor dem Fernseher überraschend. Er richtet sich damit gegen die Empfehlung seiner Mutter Cersei 
Lannister, deren strategischer Einfluss auf ihn bisher unumstritten war. Auch seiner zukünftigen Ehefrau nimmt er damit den Vater und schafft sich so eine Feindin im nahen Umfeld. Ein strategisches Motiv im Sinne eines Machterhalts kann daher bezweifelt werden. Vielmehr scheint die Hinrichtung für ihn eine willkommene Demonstration seiner Stärke und Grausamkeit zu sein, die nicht nur ihm sondern auch dem vor der Bühne stehenden johlenden Publikum sichtlich Freude bereitet.

\section{2 «The Rains of Castamere» (S3, E9)}

In dieser Episode steht eine Hochzeit im Mittelpunkt, die später als "Red wedding” im kollektiven Gedächtnis der Rezipienten und Serienfiguren verbleiben wird. Lord Frey, Lord Bolton und Tywin Lannister bilden ein Komplott gegen die miteinander verwandten Häuser Stark und Tully und locken diese in eine Falle. Unter dem Vorwand einer Hochzeit empfängt Lord Frey die Starks und die Tullys gastfreundlich und garantiert ihnen damit implizit Schutz und Sicherheit. Die anfängliche Vorsicht, die von Hauptcharakteren sowie Zuschauenden geteilt wird, schwindet allmählich, und die gute Stimmung auf der Hochzeit vermittelt Freude und Sicherheit. Robb Stark, der älteste Sohn Ned Starks und nach dessen Tod Lord von Winterfell, und seine Frau Talisa erwarten ein Kind und schmieden Zukunftspläne. Seine Mutter Catelyn Stark scheint von dem Glück ihres Sohnes erfüllt. Doch plötzlich werden die Türen des Saals von Soldaten verschlossen und Streichmusik (“The Rains of Castamere”) setzt ein. Catelyns Blick verrät, dass sie beunruhigt ist und auch die Zuschauerinnen merken, dass etwas nicht stimmt. Lord Frey setzt zu einer Rede an, in der er von einem nachträglichen «wedding gift» für Talisa und Robb spricht. Die Maske fällt und die Soldaten greifen die Hochzeitsgäste mit Messern an. Als erstes wird Robb Starks Frau Talisa getötet, indem ihr mehrfach in den schwangeren Unterleib gestochen wird, dann folgt ein Bolzenhagel, bei dem weitere Gäste ermordet werden. Der Schock bei den Zuschauenden wird von einem Funken Hoffnung begleitet, da die Hauptcharaktere Catelyn und Robb Stark beide noch am Leben sind. Während erstere unter einem Tisch Schutz sucht, schleppt sich letzterer zu seiner toten Frau. Catelyn schnappt sich die Frau von Lord Frey und droht, ihr die Kehle durchzuschneiden, wenn er ihren Sohn Robb nicht gehen lässt. Sie bittet und bettelt ("let it end, please [...] he is my son, my first son”) und noch einmal wird die Hoffnung der Zuschauenden geschürt, dass beide (oder zumindest eine(r)) überleben könnte(n). Nach einer kurzen Pause erwidert Lord Frey: “I'll find another.” Dann wird Rob vor den Augen seiner Mutter erstochen, und Catelyn, die ihre Geisel teilnahmslos tötet, wird ebenfalls die Kehle durchgeschnitten. 
Ähnlich wie im Falle Ned Starks sind es auch hier lieb gewonnene Hauptfiguren, die dem Zuschauer auf grausame Art und Weise genommen werden. Und abermals entsteht der Eindruck, dass mit dem Sicherheitsgefühl der Zuschauenden gespielt wird, indem immer wieder Hoffnung geschürt und dann wieder zerstört wird. Die Verschwörer haben für ihre Taten zwar Motive, die sich als machtpolitisch zusammenfassen lassen, allerdings überschreiten sie eine wichtige soziale Norm, indem Lord Frey das in Westeros geltende «Gastrecht» (dem Gast wird Schutz zugesichert) bricht. Es sind in dieser Folge also sowohl die Serienmacher, als auch die Figuren innerhalb der Serie, die ungeschriebene Regeln missachten, ergo enthemmt agieren. Somit wird abermals das Motiv der fehlenden Sicherheit bemüht und um einen Punkt erweitert: Niemand ist sicher vor niemandem und auch geltende Normen bieten keinen Schutz.

\section{3 «The Mountain and the Viper» (S4, E8)}

Diese Episode, die aufgrund ihres grausamen Finales vielfach rezipiert wurde, stellt ein Duell in den Mittelpunkt. Hintergrund dessen ist die Beschuldigung Cersei Lannisters, ihr Bruder Tyrion Lannister habe ihren Sohn/seinen Neffen vergiftet und damit dessen Tod verschuldet. Da dieser die Vorwürfe bestreitet, soll ein Kampf über die Schuld Tyrions entscheiden. Beide Parteien wählen einen Champion, der an ihrer Stelle in den Ring steigen soll. Während Cersei den ihr treu ergebenen und für seine Blutrünstigkeit bekannten Gregor Clegane alias The Mountain wählt, erklärt sich schliesslich der kampferprobte Oberyn Martell alias The Viper bereit, diese Aufgabe für Tyrion zu übernehmen (E7). Er sieht in dem Duell die Chance, seine im Auftrag von den Lannisters getötete Schwester zu rächen. In der Arena beeindruckt Oberyn Martell das Publikum mit siegessicherem Auftreten und Demonstrationen seiner Kampfeskunst. Er fordert The Mountain auf, den Mord an seiner Schwester zu gestehen: "I am going to hear you confess before you die. You raped my sister. You murdered her. You killed her children.” Sätze, die Martell während des Kampfes stetig wiederholt. Er verletzt The Mountain, der daraufhin zu Boden geht und versetzt ihm mit seinem Speer einen vermeintlich tödlichen Stich in die Brust. Den Mienen des Publikums ist anzusehen, dass dieses den Kampf nun für beendet hält, da zieht Oberyn Martell den Speer jedoch wieder aus der Brust seines Kontrahenten (“No, no, no, you can’t die yet. You haven’t confessed!”) Oberyn Martell riskiert einen Blick zu seiner Geliebten, eine kurze Unaufmerksamkeit, die The Mountain nutzt, um ihn zu überwältigen. Er drückt dem nun unter ihm liegenden Martell die Augen in den Kopf und zerquetscht ihm den Schädel. Anstelle des Kopfes ist nur noch eine riesige Blutlache zu sehen. 
Dieser Handlungsstrang kontrastiert die Frage nach dem Motiv in Form eines Duells, da hier ein Kämpfer mit gegen einen Champion ohne eigene Beweggründe antritt; während Oberyn Martell das Duell als Chance sieht, den Tod seiner Schwester zu rächen, ist sein Gegner The Mountain nur das Instrument für die Rachegelüste Cersei Lannisters. Schlussendlich kostet Oberyn Martell aber genau das Vorhandensein dieses Motivs den Sieg. Neben dem Schock, dass letztendlich nicht er, sondern The Mountain das Duell gewinnt, das Böse also (wieder mal) obsiegt, ist es zusätzlich die archaische Brutalität, die sowohl das Publikum auf der Tribüne, als auch jenes auf dem Sofa schockiert zurücklässt.

Die bisher genannten Szenen sollten die Grausamkeit und Enthemmtheit von GoT exemplarisch veranschaulichen. Hinzu kommt, dass Brutalität und Grausamkeit in der Serie nicht auf einzelne, wenige oder spezifische Szenen beschränkt bleiben, sondern ein fester Bestandteil der gesamten Serie sind. Neu ist dabei unseres Erachtens nicht nur die beschriebene Enthemmung im Sinne des Ausmasses an Grausamkeit, sondern auch die Zielpersonen. So werden Kinder in GoT regelmässig Opfer grausamer Rituale oder anderweitiger Machtdemonstrationen: Die spätere Armee von Daenerys Targaryen besteht aus sogenannten «unberührbaren» Kämpfern, die als kleine Kinder bereits ihren Eltern weggenommen und unter unmenschlichen Bedingungen (einschliesslich ihrer Kastration) zu Kampfmaschinen ausgebildet wurden. Um sich als in diesem Sinne erfolgreich ausgebildeter Soldat zu beweisen und die gewünschte Abwesenheit jeglicher Gefühle zu demonstrieren, muss jeder von Ihnen - als Mutproben-Aufnahmeritual - einer Mutter einen Säugling aus dem Arm reissen und diesen vor ihren Augen töten. Daenerys 8000 Mann starke Armee bedeutet vor diesem Hintergrund also 8000 tote Säuglinge. Auf dem Weg nach Mereen kommt Daenerys Gruppe an einem Kreuz vorbei, an dem ein totes Kind hängt, mit dem der Weg in die Hauptstadt angekündigt wird, insgesamt folgen noch 162 gekreuzigte Kinder. Robert Baratheons unehelicher Sohn, ein kleines Baby, wird ebenfalls vor den Augen seiner Mutter von Soldaten getötet. Auch Stannis Baratheons Tochter Shireen landet schließlich auf Weisung der Priesterin Melisandre als Menschenopfer auf dem Scheiterhaufen mit Einverständnis ihres Vaters. Die Zuschauenden hören ihre Todesschreie und teilen ihr Entsetzen mit dem ihrer Mutter, die vorher zwar kaltherzig und schroff, aber zumindest in diesem Moment anders als ihr Ehemann zu einer adäquaten Reaktion fähig ist. Diese Szenen sind unserer Ansicht nach die überzeugendsten Belege dafür, warum bei der Gewalt in GoTvon einer enthemmten Gewalt gesprochen werden kann, weil sie die Zuschauenden zuhause oftmals fassungslos zurücklässt. Die Darstellung von Enthemmung findet dabei auf zwei Ebenen statt: Zum 
einen bewegt sie sich auf einem expliziten Level der körperlichen Brutalität, zum anderen auf einem impliziten Level der Motivlosigkeit. Beide sollen im Folgenden näher erläutert werden.

Hinrichtungen in Form einer Köpfung, ein zerstochener Babybauch, sich wiederholende Folterszenen und ein platzender Schädel sind nur wenige Beispiele für die explizite Form der Gewalt, die die Serienmacher in GoT darstellen. Dass die Gewaltdarstellungen nicht nur Selbstzweck im Sinne höherer Einschaltquoten ("violence sells") sind, zeigen die intertextuellen und metaphorischen Bezüge innerhalb der Serie, die mit den Gewaltdarstellungen in Verbindung stehen. Dabei ist vor allem die Episode The Mountain and the Viper hervorzuheben. Tyrion Lannister führt, eingesperrt im Kerker, ein vielleicht letztes Gespräch mit seinem Bruder Jamie Lannister. Ihr Gespräch fällt auf ihren Cousin Orson Lannister, der den ganzen Tag nichts anderes tat, als Käfer mit einem Stein zu zerquetschen. Folgt man Poscheschnik (2016), der für eine «Offenlegung der latenten Sinnstruktur von medial transportierten Narrativen und Inszenierungen» (S. 11) plädiert, lässt sich dieses Gespräch nicht nur als Vorbote für das sich anbahnende Unheil verstehen, sondern in Verbindung mit den weiteren Ereignissen dieser Episode (z. B. Ermordung von Dorfbewohnern durch die Wildlings), auch als eine Erzählung über Macht und Ohnmacht. Es sendet eine düstere sozialdarwinistische Botschaft von einer enthemmten Welt, in der Menschen andere Menschen wie Käfer zerquetschen, unabhängig davon, ob sie ein Motiv dafür haben oder nicht. Der Mangel an Motiven führt dazu, dass das Handeln der Täter willkürlich und somit unberechenbar erscheint, was bei den Zuschauenden ein starkes Gefühl von Unsicherheit hervorruft. Dieses wird von den Serienmachern durch dramaturgische Techniken verstärkt; so folgen die Handlungen meist einem Muster: auf die wahrgenommene Bedrohung folgt eine kurzfristige Entschärfung der Situation, in der die Hoffnung auf ein gutes Ende keimt, gefolgt von der Zerstörung dieser Hoffnung durch einen dramatischen Ausgang. Der zweite Schritt sorgt dafür, dass die Fallhöhe wie in der aristotelischen Tragödie gesteigert und somit der Ausgang als noch dramatischer wahrgenommen wird. Zudem befinden sich die bereits angesprochenen «Vorboten» des sich anbahnenden Unheils in jeder der besprochenen Episoden. In der Folge «Baelor» (S1, E9) lässt sich die Taube, die Arya fängt, als solcher verstehen, während es bei “The Rains of Castamere” (S3, E9) das gleichnamige Lied der Familie Lannister ist, das auf einen Sieg dieser hindeutet. Gemein ist diesen Vorboten, dass sie sich erst nach Vollzug der grausamen Handlung als solche entschlüsseln lassen.

Bei allen hier besprochenen Episoden gibt es neben einem Publikum, das zuhause vor dem Bildschirm sitzt, und das nach Storck als extradiegetisches 
Publikum zu bezeichnen ist, auch eine Zuschauerschaft, die den Szenen innerhalb der Serie beiwohnt, das intradiegetische Publikum (Storck, 2017, S. 26 f.). So ist letzteres bei Ned Starks Hinrichtung das Volk aus Kings Landing, bei der Red Wedding die Hochzeitsgesellschaft, und bei dem Duell die Zuschauer im Amphitheater. Die intradiegetischen Zuschauer/innen sind passiv, greifen also nicht in das Geschehen ein, lassen sich aufgrund ihrer Reaktion allerdings zwei unterschiedlichen Kategorien zuordnen. Die erste Kategorie könnte man mit «unlustvolle Betrachter» überschreiben, die mit ihrer Reaktion (Lähmung, Schock) die Gefühle des extradiegetischen Publikums spiegeln. Die Hochzeitsgesellschaft der Red Wedding und die Zuschauenden beim Duell würden hierein fallen. Die Gefühle der zur zweiten Kategorie zugehörigen «lustvollen Beobachter/innen» hingegen stehen denen der Rezipienten diametral entgegen. Bei der Hinrichtung von Ned Stark reckt das Volk die Fäuste in die Höhe und feiert den König Joffrey für seine Entscheidung, den «Verräter» köpfen zu lassen.

Die generelle Passivität des intradiegetischen Publikums steht für das Wissen um ihre Hilflosigkeit und Machtlosigkeit. Es eint sie die feste Überzeugung, dass sie nichts tun können, um das anbahnende Geschehen aufzuhalten. Die einzigen Freiheitsgrade ergeben sich bezüglich ihrer Reaktion auf das Grausame. Während die einen also gelähmt oder anders ausgedrückt gehemmt reagieren, erleben die anderen durch die Identifikation mit den Tätern lustvolle Enthemmung. Hier werden also zwei Seiten derselben Medaille gezeigt, in einer Spaltung, die so möglicherweise bei den Zuschauenden daheim gar nicht existiert. Wir dürfen vermuten, dass beide Gefühle vorhanden sind: Ein gleichzeitig Angezogen- und ein Abgestossen-sein von dieser primitiven und archaischen Form der Gewalt. Wir werden darauf noch einmal zurückkommen.

\section{GoT als Erkundung der Bedingungen menschlicher Moral}

Der Wegfall des Production Code hat, so viel scheint sicher, zu einer deutlichen Zunahme an visualisierter Gewalt innerhalb der Film- und FernsehserienLandschaft geführt, die fortzuschreiten scheint: Gegenüber den Szenen in GoT wirken die Gewaltszenen aus The Wild Bunch fast harmlos.

Wenn nach Winter Serien als «Produkte der Populärkultur kulturelle und gesellschaftliche Konstellationen und Kontexte artikulieren und ihre Komplexität gewinnbringend für die gesellschaftliche Auseinandersetzung analysieren können» (Winter, 2015b, S. 380), dann lässt sich fragen, auf welche gesellschaftlichen Veränderungen die zeitgenössischen Serien mit dieser von uns konstatierten Enthemmung, bzw. dem starken Fokus auf primärprozesshaftem Geschehen reagieren. 
Winter (2015a) konstatierte bereits für Filme wie The Wild Bunch oder Bonnie and Clyde, dass diese mit ihren Gewaltdarstellungen auf die kulturellen und sozialen Veränderungen der 60er Jahre reagierten, womit die Berichterstattung über den Vietnamkrieg gemeint war, aber auch die politischen Attentate auf die Kennedys, Martin Luther King und Malcom X, die Lynchmorde durch die MansonBande, sowie das Anwachsen der Strassenkriminalität in den US-amerikanischen Grossstädten (ebd., S. 160-161). So habe Peckinpah in einem Interview 1969 gesagt: «Violence is ugly, brutalizing und bloody fucking awful. It's not fun and games and cowboys and Indians, it's a terrible, ugly thing» (zit. nach Winter, 2015a, S. 161).

Und als solches hat er auch seine Filme inszeniert. Etwas Ähnliches hat der Autor der Romanvorlage von GoT George R. R. Martin auf die Kritik an der zunehmenden sexualisierten Gewalt in der Serie geantwortet:

Vergewaltigungen und sexuelle Gewalt sind Teil eines jeden Krieges, der jemals geführt wurde, von den alten Sumerern bis heute. Diese Aspekte bei einer Geschichte über Krieg und Macht auszulassen wäre falsch und unehrlich. (Martin in einer E-Mail an die New York Times, zitiert nach Schmieder, 2014)

In dieser Logik liesse sich freilich jede Form von enthemmter Gewalt und Brutalität rechtfertigen: Die Welt ist schlecht, oder, um mit Horkheimer und Adorno zu sprechen: «Die vollends aufgeklärte Erde strahlt im Zeichen triumphalen Unheils» (Horkheimer \& Adorno, 1947, S. 19), und konsequenterweise muss dies auch medial verarbeitet werden. Die heute ständig neu entstehenden Kriege, Hungerkatastrophen und die Ausbreitung des islamischen Staats bezeugen die Wahrheit und Aktualität dieser Aussage. Dennoch lässt sich fragen, ob eine zunehmend brutale Darstellungsweise im TV diese Gewalt nicht eher reproduziert, denn wirklich hinterfragt und kritisiert.

Der Tod Ned Starks, der die meisten Zuschauerinnen so schockiert hat, dass er in unzähligen Foren diskutiert und in Zeitungsartikeln besprochen wurde, sogar manche Fans dazu inspiriert hat, einen Videoclip zu drehen, in dem sie ihren Schock über seinen Tod verarbeiten², dieser Tod also, der nach Storck als «"game changer” (2017, S. 23) in der Logik zeitgenössischer Fernsehserien betrachtet werden kann, spiegelt aber möglicherweise ein Lebensgefühl wieder, das seit dem 11. September 2001 die westlichen Gesellschaften weit tiefer erfasst hat, als sie sich eingestehen wollen. Dieses Ereignis markiert eine Zäsur, weil nämlich etwas passiert war, was vorher nie für möglich gehalten wurde: Die westliche Welt, die Supermacht USA 
wurde angegriffen. Das Gefühl: Niemand ist sicher, das sich beim Gucken von GoT von der ersten Staffel an ausbreitet, wurde zunehmend auch ein Lebensgefühl in den westlichen Industriegesellschaften nach 9/11 und dem sich seither stetig ausbreitenden islamischen Staat, der in regelmässigen Abständen Bombenattentate in europäischen Grossstädten plant und verübt. Visuell werden durch das Internet Enthauptungsvideos verbreitet, und die Medien sparen nicht mit Berichten über die Gräueltaten des IS. So gesehen bedient GoT beides: es nimmt einerseits ein Lebensgefühl auf und macht es andererseits durch die mediale Inszenierung einer (psychischen) Bearbeitung oder zumindest geteilten Erfahrung (wie in den von Storck besprochenen reaction videos, Storck, 2017, S. 13 ff.) zugänglich.

Wenn ein Journalist namens Kashoggi nichtsahnend und sich in Sicherheit wiegend in eine Botschaft eines - sich zumindest nach aussen hin rechtstaatlich und demokratisch gebenden - Landes wie der Türkei geht, um sich seine Hochzeitspapiere abzuholen, dann dort gefoltert und lebendig zerteilt wird, so erinnert diese Grausamkeit und Hinterhältigkeit in erschreckender Weise an die oben beschriebene Szene der red wedding aus GoT. Auch hier gibt es ein klares machtpolitisches Motiv, die Grausamkeit und enthemmte Agierweise wird dadurch jedoch nicht erklärt.

In Anlehnung an Storck (2017) bzw. Robert Pfaller (2008) könnte man die These aufstellen, dass es beim Konsumieren von GoTinterpassiv eineVerschränkung von Lust an der Unlust/dem Ekel, dem Schockiertsein gibt und eine Unlust und ein Empörtsein angesichts der scheinbar in GoTgenossenen Lust an der Grausamkeit. Die Zuschauer/innen geniessen, bzw. erleiden diese Gewalt sekundär vermittelt über Identifikation entweder mit dem Aggressor oder dem Opfer. Die Ausführung selbst wird an die TV-Serienakteure delegiert. Storck hat in diesem Zusammenhang zurecht die Verbindung zu Robert Pfallers Theorie der Interpassivität gezogen, in der «die Konsumtion eines Produkts von den Konsumenten zu einer stellvertretenden Konsumtions-Instanz verlagert» wird (zit. nach Storck 2017, S. 21). So gesehen könnte das, was Storck mit Pfaller für das Geniessen konstatiert, auch für die Aggression gelten:

Doppeldeutig ist das delegierte Genießen, weil es immer eine Entwertung und Verachtung dessen, der an meiner Stelle genießt, und damit auch der (eigenen, delegierten) Lust gibt. So entsteht eine Figur der Überkreuzung. Interpassiv genießend erlebe ich Lust an meiner Unlust und Unlust angesichts meiner Lust. (Storck 2017, S. 22) 
Wir unterstellen, dass es auf Zuschauerinnen-Seite neben der Verachtung und dem Ekel gegenüber der gezeigten und ausgeführten Aggression auch eine (delegierte und abgewehrte) Lust-Seite gibt, die sich interpassiv überkreuzt und speziell in GoT durch das dort bei den Gewaltszenen häufig anwesende Publikum noch einmal verdoppelt wird (vgl. ebd., S. 27).

Auch wenn eine ganze Reihe anderer zeitgenössischer Serien implizit ebenfalls der Frage nachgeht, wie soziale Strukturen sich bilden oder neubilden (und ob), wenn äussere Ordnungsstrukturen wie Gesetz oder Exekutive nicht etabliert sind (u. a. The Walking Dead, Deadwood, Hell on Wheels), so nimmt GoT in dieser Reihe doch eine besondere Stellung ein, insofern sich die Serie, vermittelt über die Besonderheiten, die sich in der Rezeption zeigen, noch einmal in besonderer Weise (hinter)fragt, wie sich Individuen verhalten, wenn die Hemmung durch internalisierte Strukturen (Über-Ich, Sekundärprozess) ausser Kraft gesetzt wird.

Nimmt man die Annahme ernst, dass zeitgenössische (TV-)Serien ein Medium darstellen, in dem gesellschaftliche Prozesse analysiert werden können, dann liegt die Folgerung nah, dass in GoT (ohne dass dies bewusst intendiert sein müsste) ein Versuch zu sehen ist, menschliche Gewalt und Amoralität zu verstehen - denen wir teils eben in Nachrichtensendungen (wie der Meldung von dem Tod des Journalisten Kashoggi) nicht weniger drastisch begegnen und die uns ebenso schockiert zurücklassen wie die oben besprochenen Szenen aus GoT.

GoT markiert diese menschliche Gewalt und Amoralität und spitzt sie zu, so dass schliesslich die Frage aufgeworfen werden kann, was es für die Internalisierung von Strukturen bedarf, die Menschen zur Moralität befähigen. Äussere und damit auch internalisierte elterliche Objekte haben in GoT jedenfalls oft keine gute Rolle - es gibt Infantizid, Inzestkinder, Tod im Kindesbett, uvm. Und auch die Beziehungen, die zunächst nicht unmittelbarer Triebbefriedigung dienen oder instrumentell motiviert sind, sondern in denen zunächst die Bindung und zwischenmenschliche Beziehung im oben thematisierten sekundärhaften Prozess im Vordergrund zu stehen scheinen (z. B. die inzestuöse Liebesbeziehung zwischen den Geschwistern Jamie und Cersei Lannister oder Tyrions Liebesbeziehung zu der Prostituierten Shae), kippen nach einiger Zeit und die destruktiven Elemente, bzw. primärprozesshafte Reaktionen gewinnen in Form von Vergewaltigung (Jamie und Cersei) und Mord (Tyrion und Shae) wieder die Oberhand. Einzig die (Patchwork-) Kleinfamilie um die Figur des Samwell Tarley scheint hier eine Ausnahme darzustellen. Bislang jedenfalls wurden er, seine Frau Goldy und sein Ziehsohn sowohl vom Autor als auch den Serienmachern «verschont», und auch untereinander scheinen die Beziehungen intakt. Sam wird zudem als Intellektueller dargestellt, 
der sein Wissen mit seiner Frau teilt und sie zum Lesen und zur Bildung motiviert, wodurch auch ein anderer machtheoretischer Zugang (auch Wissen ist Macht) sichtbar wird. Möglicherweise scheint hier in dieser «heilen Kleinfamilie» eine Utopie von nicht enthemmten zwischenmenschlichen Beziehungen auf, inmitten einer ansonsten amoralischen Welt. So gesehen hätte die in GoT dargestellte und gelebte Enthemmung möglicherweise nicht nur schlicht Unterhaltungswert, wäre Ausdruck zeitgemässen Empfindens einer Unsicherheit oder (nach Marcuse) primärer Impulse nach Aggressivität, sondern die Serie könnte auch (ohne das bewusst intendiert zu haben) durch das Mittel der drastischen Darstellung bis hin zur Übertreibung die Möglichkeit eröffnen, darüber zu reflektieren, wie und unter welchen Bedingungen moralisches Handeln und ethisches Empfinden (z. B. verkörpert durch Samwell Tarley) sich realisieren kann. Dafür braucht es die Hemmung, hervorgerufen durch das Über-Ich als Internalisierung vormals äusserer Verbote, sowie den Sekundärprozess, und andererseits die zielgehemmte triebhafte Lust, die sozialen Bedürfnissen eine Gestaltungskraft zuführt.

\section{Literatur}

Black, G. D. (1989). Hollywood Censored. The Production Code Administration and the Hollywood Film Industry, 1930-1940. Film History (3), 167-189.

Duden online (2018). Stichwort: Cliffhanger. https://www.duden.de/rechtschreibung/Cliffhanger [20.11.2018].

Eichner, S. (2013). Blockbuster Television. In S. Eichner, L. Mikos \& R. Winter (Hg), Transnationale Serienkultur. Theorie, Ästhetik, Narration und Rezeption neuer Fernsehserien (S. 45-65). Wiesbaden: Springer.

Evans, D. (1996). Wörterbuch der Lacanschen Psychoanalyse. Wien 2002: Turi + Kant.

Freud, S. (1900a). Die Traumdeutung. GW II/III.

Freud, S. (1911b). Formulierungen über die zwei Prinzipien des psychischen Geschehens. GWVIII, S. 229-238.

Freud, S. (1915c). Triebe und Triebschicksale. GWX, S. 209-232.

Freud, S. (1915e). Das Unbewusste. GWX, S. 263-303.

Freud, S. (1921c). Massenpsychologie und Ich-Analyse. GW XIII, S. 71-161.

Freud, S. (1926d). Hemmung, Symptom und Angst. GW XIV, S. 111-205.

Freud, S. (1930a). Das Unbehagen in der Kultur. GW XIV, S. 419-506.

Freud, S. (1950a). Entwurf einer Psychologie. GW Nachtragsband, S. 373-486.

Gormász, K. (2015). Walter White \& Co.: Die neuen Heldenfiguren in amerikanischen Fernsehserien. Köln: Halem. 
Greene, J. M. (2010). Hollywood's Production Code and Thirties Romantic Comedy, Historical Journal of Film, Radio and Television, 30 (1), 55-73.

Hamburger, C. 18.09.2015. Fargo, Hannibal, Scream:Wenn Kinofilme in Serie gehen. Augsburger Allgemeine, https://www.augsburger-allgemeine.de/panorama/ Fargo-Hannibal-Scream-Wenn-Kino-Filme-in-Serie-gehen-id35523137.html [11.11.2018].

Horkheimer, M. \& Adorno, T.W. (1947; 1997). Dialektik der Aufklärung. In T.W. Adorno (Hg), Gesammelte Schriften Bd. 3 (S. 7-336). Darmstadt: Wissenschaftliche Buchgesellschaft.

Kalle, M. 08.03.2018. Glück in Serie. ZeitMagazin, https://www.zeit.de/zeit-magazin/ 2018/11/fernsehserien-the-sopranos-breaking-bad-the-wire-kultur.

Lexikon der Filmbegriffe (2012). Spinoff. Kiel: Universität Kiel. http://filmlexikon. uni-kiel.de/index.php?action=lexikon\&tag=det\&id=2752 [12.12.2018].

Lexikon der Filmbegriffe (2012). Prequel. Kiel: Universität Kiel. http:/ filmlexikon. uni-kiel.de/index.php?action=lexikon\&tag=det\&id=295 [12.12.2018].

Marcuse, H. (1956;1967). Aggressivität in der gegenwärtigen Industriegesellschaft. In H. Dahmer (Hg) (2013), Analytische Sozialpsychologie Bd. 2 (S. 452-470). Gießen: Psychosozial Verlag.

Metelmann, J. (2008). Gewalt im Film. In M. Schroer (Hg), Gesellschaft im Film (S. 111-128). Konstanz: UVK.

Nesselhauf, J., Schleich, M. (2015). Vorwort. Feeling that the best is over. Vom Ende der Qualität und der Qualität von Enden. In J. Nesselhauf, M. Schleich (Hg), Das andere Fernsehen?! Eine Bestandsaufnahme des «Quality Television» (S. 9-31). Bielefeld: transcript Verlag.

Pfaller, R. (2008). Ästhetik der Interpassivität. Hamburg: Philo Fine Arts.

Poscheschnik, G. (2016). Game of Thrones - Fernsehserien als Artikulation gesellschaftlich-unbewusster Phantasien. Medien Pädagogik (26), 1-12.

Schmieder, J. (2014). Zu viel Suff, Sex und Mord. Süddeutsche Zeitung, https: / /www. sueddeutsche.de/medien/game-of-thrones-in-der-kritik-zu-viel-suff-sexund-mord-1.1949585 [14.01.2019].

Schröder, J. (2017). «Game of Thrones» bricht in den USA alle Zuschauerrekorde auch dank Streaming. Media, https://meedia.de/2017/08/29/game-ofthrones-bricht-in-den-usa-alle-zuschauerrekorde-auch-dank-streaming/ [14.01.2019].

Storck, T. \& Billhardt, F. (in Vorb.). Denken, Wahrnehmung, Gedächtnis. Psychoanalyse und Allgemeine Psychologie. Stuttgart: Kohlhammer. 
Storck, T. (2017). Game of Thrones. In T. Storck \& S. Taubner (Hg), Von Game of Thrones bis The Walking Dead (S. 11-29). Deutschland: Springer-Verlag.

Thompson, J. R. (1996). From Hill Street Blues to ER. Television's Second Golden Age. New York: Syracuse University Press.

Timmer, J. (2011). Restricting Portrayals of Film Violence to Reduce the Likelihood of Negative Effects in Viewers: Did the Framers of the Motion Picture Production Code Get It Right? Journal of Popular Film \& Television, 39 (1), 29-36.

Winter, M., Storck, T. \& Müller-Hermann, A. (2018). Mutterschaft in zeitgenössischen TV-Serien am Beispiel der Serien Homeland. In H. Krüger-Kirn \& L. Wolf (Hg), Mutterschaft zwischen Konstruktion und Erfahrung. Aktuelle Studien und Standpunkte (S. 105-118). Opladen: Barbara Budrich.

Winter, R. (2013). Fernsehserien als Kult. In S. Eichner, L. Mikos \& R. Winter (Hg), Transnationale Serienkultur: Theorie, Ästhetik, Narration und Rezeption neuer Fernsehserien. (S. 67-83). Wiesbaden: Springer.

Winter, R. (2015a). Let's Go! Gewalt, Gesetz und Erlösung in The Wild Bunch. In A. Keppler, J.-F. Popp \& M. Seel (Hg), Gesetz und Gewalt im Kino (S. 156-175). Frankfurt/ New York: Campus.

Winter, R. (2015b). Review: The Wire. Analysen zur Kulturdiagnostik populärer Medien. (Ahrens, J., M. Cuntz, L. Koch, M. Krause und P. Schulte 2014, Wiesbaden:VS Verlag) Kölner Zeitschrift für Soziologie und Sozialpsychologie 67 (2), 379-382

Zielcke, A. 13.02.1995. Das Ende der Apokalypse. Der Spiegel, http://www.spiegel. de/spiegel/print/d-9158181.html [15.11.2018].

\section{Angaben zu den Autorinnen und Autoren}

Dr. Merve Winter (Dipl.-Psych.) arbeitet als wissenschaftliche Mitarbeiterin an der Psychologischen Hochschule Berlin (PHB) im Arbeitsbereich Klinische Psychologie mit dem Schwerpunkt Tiefenpsychologie (Prof. Storck). Ferner arbeitet sie als Psychoanalytikerin in Ausbildung am IfP. Für das Universitätsklinikum Leipzig ist sie zudem als psychologische Gutachterin für Lebendorganspenden tätig.

Timo Storck, Prof. Dr. phil., Professor für Klinische Psychologie und Psychotherapie an der Psychologischen Hochschule Berlin, psychologischer Psychotherapeut und Psychoanalytiker (DPV/IPA). Forschungsschwerpunkte: psychosomatische Erkrankungen, Fallbesprechungen in der stationären Psychotherapie, psychoanalytische Grundkonzepte und Methodologie, Filmpsychoanalyse, konzeptvergleichende Psychotherapieforschung. 
Jelka Berger, Masterstudentin im vierten Fachsemester an der Psychologischen Hochschule Berlin (PHB) mit Schwerpunkt Klinische Psychologie.

\section{Anmerkungen}

1 Wir werden im Folgenden in abwechselnder Reihenfolge das weibliche und männliche Geschlecht verwenden.

2 A Character I used to know - A «Game of Thrones» Season 1 Parody by Not Literally Productions: https://www.youtube.com/watch?v=ZXSSBu9uKrM 